Research Paper

\title{
Impairment of the Peritoneal Surface as a Decisive Factor for Intestinal Adhesions in Intraperitoneal Onlay Mesh Surgery - Introducing a New Rat Model
}

\author{
M Winny ${ }^{1 *}$, L Grethe ${ }^{1^{*}}$, L Maegel${ }^{2}$, D Jonigk ${ }^{2}$, T Lippmann², J Klempnauer ${ }^{1}$, D Poehnert ${ }^{1}{ }^{凶}$ \\ 1. Clinic for General, Abdominal and Transplant Surgery, Hannover Medical School, Germany; \\ 2. Institute of Pathology, Hannover Medical School, Germany. \\ "contributed equally to this work \\ $\triangle$ Corresponding author: Daniel Poehnert. poehnert.daniel@mh-hannover.de
}

() Ivyspring International Publisher. Reproduction is permitted for personal, noncommercial use, provided that the article is in whole, unmodified, and properly cited. See http://ivyspring.com/terms for terms and conditions.

Received: 2015.10.06; Accepted: 2015.12.10; Published: 2016.02.01

\begin{abstract}
Background: Meshes implanted intraperitoneally are known to cause adhesions potentially resulting in complications such as chronic pain, enterocutaneous fistula, or mesh infection. This study introduces a model for investigation of intestine-to-mesh adhesions and evaluates as to whether missing of visceral peritoneum is causative.

Methods: In 18 rats, rectangular $1.5 \times 2 \mathrm{~cm}$ patches of an uncoated polypropylene mesh (UItrapro ${ }^{\circledR}$ ) were sewn to the inner abdominal wall next to the cecum. Additionally, a meso-suture ensured contact between cecum and mesh. Rats were assigned to 2 groups: in 8 rats the peritoneum was left intact, in 10 the cecum was depleted from peritoneum with abrasion. Sacrifice was on day 7. Macroscopic evaluation used two adhesion scores. Specimens were evaluated microscopically, statistical analyses employed student's t-test.

Results: On day 7, rats with mesh implantation combined with locally de-peritonealization by cecal abrasion mostly showed severe cecum-to-mesh agglutination (mean Lauder score $92 \%$, mean total Hoffmann score $90 \%$ ), whereas meshes of most animals without cecal abrasion only had some coverage with intraabdominal fat $(33 \%, 24 \% ; p=0.0002)$. Histological work-up showed adequate wall ingrowth of mesh in all rats. In animals with cecal abrasion, meshes were mostly adhesive with cecal wall. However, when the peritoneum of cecum was unimpaired, abdominal wall above the mesh as well as cecum usually revealed sub-peritoneal tissue and a mono-layer cell coverage as seen in normal peritoneum.

Conclusion: This study introduces a model mimicking a clinical situation of e.g. hernia repair by intraperitoneally implanted meshes when mesh has contact with normal and with de-peritonealized intestine. The model might be useful for testing mesh types and coatings as well as other devices for their efficacy in adhesion prevention. The high adhesion scores of rats with local de-peritonealization compared with the low scores of animals with intact peritoneum indicate that the integrity of intestinal peritoneum is a decisive factor for adhesion formation.
\end{abstract}

Key words: Hernia mesh repair, intraperitoneal onlay mesh surgery, Polypropylene mesh, Adhesion formation, Experimental study, Cecal abrasion.

\section{Introduction}

Adhesion formation following hernia repair via mesh implantation can be a severe complication especially when using intraperitoneal onlay mesh implantation (IPOM). Sequelae of adhesion formation can be chronic abdominal pain 1-5, female infertility 1-3,6, enterocutaneous fistulas $7-11$, bowel obstruction and incarceration 2,3,10-12. Furthermore, adhesions may seriously complicate reoperations 13-16 
and generally increase the risk of redo surgery. Depending on surgical technique and type of mesh, an incidence of adhesion formation of about $30 \%$ is reported 17,18 . The variety of implants available on the market providing additional features such as coating to reduce the incidence of intestine-to-mesh adhesions underlines the relevance of this phenomenon in hernia repair.

Our recently introduced peritoneal adhesion model based on the "Harris group adhesion model" 19 provides a high reproducibility of adhesion formation 20 and is helpful to test and compare different adhesion prevention devices. The present study introduces a new variant of this model with mesh implantation in the right lower abdomen instead of creation of an abdominal wall injury to assess whether integrity and/or impairment of peritoneal surface are a trigger for formation of intestine-to-mesh adhesions.

\section{Materials and Methods}

This study was approved by the Lower Saxony State Office for Consumer Protection and Food Safety (LAVES, Hanover, Germany; approval code 13/1327). A total of 18 rats weighing 347-383 g (mean $365 \mathrm{~g} \pm 10$ g) were used for the study. All animal experiments were performed at Zentrales Tierlabor, Hannover Medical School, Germany, which provided the knowledge (housing, caretaking, etc.) to assure life quality of laboratory animals.

\section{Preoperative Preparation}

All animals had continuous access to fresh water and were fed a commercial diet ad libitum. Daily monitoring of weight, behavioural changes and any mortality using a standard observation chart (body condition scoring, GV-SOLAS, Charité - Universitaetsmedizin Berlin) assessed animals' well-being.

\section{Surgical Procedure}

A weight-dependent dose of $80 \mathrm{mg} / \mathrm{kg}$ body Ketamine and $5 \mathrm{mg} / \mathrm{kg}$ Xylazine was given to achieve general anesthesia. Absence of flexor reflexes indicated achievement of required level of narcosis for surgery. Afterwards, the abdomen was shaved and prepared with alcohol and iodine solution. Access to the abdominal cavity was gained via a $4 \mathrm{~cm}$ median laparotomy. Rectangular $1.5 \times 2 \mathrm{~cm}$ patches of an uncoated polypropylene mesh (Ultrapro ${ }^{\circledR}$ ) were sewn with a running $4 / 0$ Prolene ${ }^{\circledR}$ suture to the inner right lower abdominal wall next to the cecum in all 18 animals. In P-INTACT rats $(\mathrm{n}=8)$ the peritoneum was left intact, whereas in P-ABRASE animals $(n=10)$ the cecum was delivered and kept moist by a watery gauze swab while a dry gauze was used to gently abrade the cecal peritoneum in a standardized man- ner. Sufficient removal of visceral peritoneum was indicated by sub-serosa bleeding and creation of a homogenous surface of petechial hemorrhages over a $1 \times 2 \mathrm{~cm}$ area. A suture from the cecal meso to the lateral abdominal wall was placed in all animals to ensure that cecum and mesh stayed in contact. The abdomen was closed using two-layer closure technique by a consecutive suture.

\section{Postoperative Management}

Animals were monitored until complete awakening and kept warm with an infrared lamp after surgery. They were given novaminsulfone in a body weight adapted dose to counteract expectable pain in the immediate postoperative period. All animals were sacrificed on postoperative day 7 by carbon dioxide narcosis followed by cervical dislocation. The peritoneal cavity was immediately re-entered through an incision at a left position remote from the original laparotomy scar to avoid impairment of any potentially formed adhesions. Cecum and abdominal wall with mesh were harvested for histopathological assessment following a standard protocol.

\section{Evaluation Parameters}

Upon autopsy, photographs of the affected areas of all animals were taken for documentation. Adhesions between cecum and mesh area were evaluated macroscopically by two independent observers according to the scoring schemes by Lauder et al. ${ }^{21}$ and Hoffmann et al. ${ }^{22}$. The Lauder scoring scheme for measurement of adhesion formation takes into account number, strength, and distribution of adhesions ${ }^{21}$. Adhesions were measured and expressed as a percentage of the total mesh surface following Hoffmann's scoring scheme. This was further translated into grades $0-4$. Each animal was also evaluated for strength of adhesion formation and graded 0-3, the extent of adhesion formation was also graded 0-3. These three sub-scores were summed for a total adhesion score 22. Histopathological assessment was performed on all cecum and abdominal wall areas with mesh samples to evaluate adhesion formation and extent of inflammatory response. Both, macroscopic and microscopic observers were blinded to the study groups.

\section{Histological examination}

Samples were excised en bloc, rinsed and immersed in $4 \%$ buffered formalin. Specimens were embedded in paraffin blocks and serial sections were stained with haematoxylin \& eosin and Periodic acid-Schiff and examined by light microscopy for histological evaluation. 


\section{Statistical Analysis}

Statistical analyses were performed with GraphPad PRISM (Version 6 for Mac OS, GraphPad Software, Inc., La Jolly, USA) using student's t-test. All adhesion scores were expressed as mean with standard deviation (SD).

\section{Results}

A total of 18 animals were included in the study; none of these animals had to be sacrificed during the course of the investigations. All animals showed equitable viability as well as course of body weight (mean body weight loss on day 7: $32.3 \pm 12.4 \mathrm{~g}$ ). There was no significant difference in the loss of bodyweight between animals without and with cecal abrasion (P-INTACT: $31.4 \pm 15.7$ g, P-ABRASE: $33.1 \pm 9.8 \mathrm{~g}$; $\mathrm{p}=$ $0.78)$.

Figure 1 shows representative photographs of animals without and with cecal abrasion intraoperatively (Figures $1 \mathrm{~A}$ and $1 \mathrm{C}$ ) and on day 7 during autopsy (Figures 1B and 1D). In P-INTACT rats, meshes for the most part revealed some coverage with intraabdominal fat (Figures 1B). In one case, the small intestine was focally adherent to the mesh and in another case the cecum showed tight and planar adhesion to the mesh. In contrast, 9 of 10 animals with cecal abrasion showed massive cecum-to-mesh adhesions (Figure 1D).
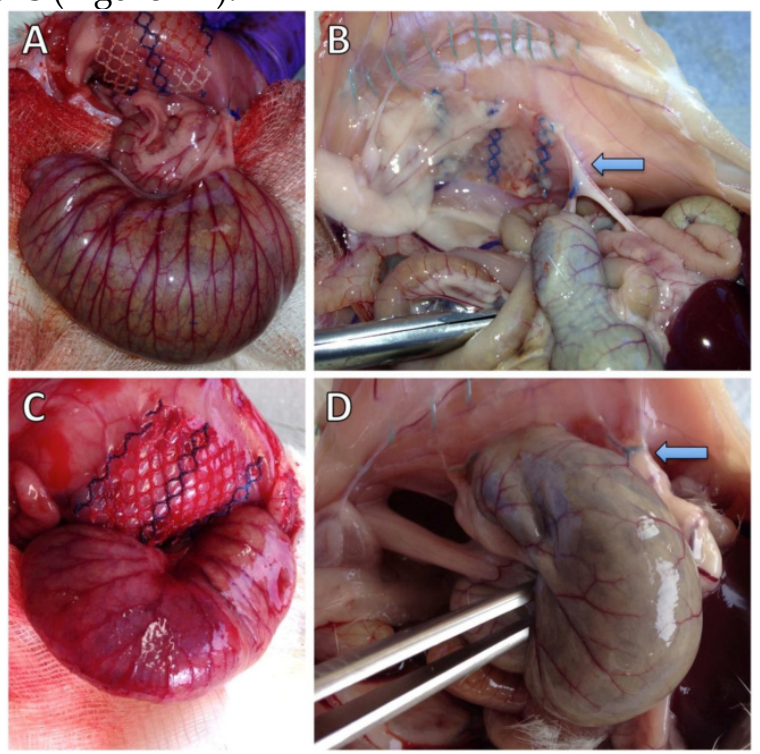

Figure 1: Representative photographs of rat cecum and abdominal wall with polypropylene mesh. A) Operative situs of a representative rat with intact cecal peritoneum (P-INTACT) after fixation of mesh to the abdominal wall. Unimpaired cecum approximated with meso-suture. (B) Situs of a representative rat with intact cecal peritoneum (P-INTACT) during autopsy on day 7 shows intraabdominal fat strands adhesive to the outer parts of the polypropylene mesh; arrow: meso-suture. (C) Operative situs of a representative rat with peritoneal abrasion of the cecum (P-ABRASE) after fixation of mesh to the abdominal wall. Abraded cecum approximated with meso-suture. (D) Situs of a representative rat with abraded cecal peritoneum (P-ABRASE) during autopsy on day 7 with the cecum adherent via mesh with the abdominal wall with maximum adhesion scoring; arrow: meso-suture.
Macroscopic inspection of mesh ingrowth into the abdominal wall revealed a good integration in both groups without and with cecal abrasion. The impact of cecal abrasion on formation of intestine-to-mesh adhesions was evaluated by comparing scores as described above. Absolute score values were further translated into percentage of respective maximum scores (Table 1).

Rats with cecal abrasion developed significantly $(p=0.0002)$ more cecum-to-mesh adhesions than animals without: mean Lauder score $4.6 \pm 1.3(=92 \%)$ and mean total Hoffmann score $9 \pm 2.8(=90 \%)$ vs. 1.6 $\pm 1.4(=33 \%)$ and $2.4 \pm 3.2(=24 \%)$. In animals without adhesions the central parts of the mesh macroscopically revealed a shiny surface comparable to unaffected peritoneum. Intraabdominal fat-to-mesh adhesions occurred predominantly at the edges of the mesh.

Table 1: Lauder and total Hoffmann scores (absolute and percentaged) of all animals.

\begin{tabular}{|c|c|c|c|c|}
\hline & \multicolumn{2}{|c|}{ Lauder score max. 5pts. } & \multicolumn{2}{|c|}{ Total Hoffmann score max. 10pts } \\
\hline & score & [\%] & score & [\%] \\
\hline \multicolumn{5}{|c|}{ P-INTACT } \\
\hline A1 & 1 & $20 \%$ & 1 & $10 \%$ \\
\hline $\mathrm{A} 2$ & 1 & $20 \%$ & 1 & $10 \%$ \\
\hline A3 & 1 & $20 \%$ & 1 & $10 \%$ \\
\hline A4 & 5 & $100 \%$ & 10 & $100 \%$ \\
\hline A5 & 1 & $20 \%$ & 1 & $10 \%$ \\
\hline A6 & 1 & $20 \%$ & 1 & $10 \%$ \\
\hline A7 & 1 & $20 \%$ & 1 & $10 \%$ \\
\hline A8 & 2 & $40 \%$ & 3 & $30 \%$ \\
\hline \multicolumn{5}{|c|}{ P-ABRASE } \\
\hline B1 & 5 & $100 \%$ & 10 & $100 \%$ \\
\hline B2 & 5 & $100 \%$ & 10 & $100 \%$ \\
\hline B3 & 5 & $100 \%$ & 10 & $100 \%$ \\
\hline B4 & 5 & $100 \%$ & 9 & $90 \%$ \\
\hline B5 & 5 & $100 \%$ & 10 & $100 \%$ \\
\hline B6 & 5 & $100 \%$ & 10 & $100 \%$ \\
\hline B7 & 5 & $100 \%$ & 10 & $100 \%$ \\
\hline B8 & 1 & $20 \%$ & 1 & $10 \%$ \\
\hline B9 & 5 & $100 \%$ & 10 & $100 \%$ \\
\hline B10 & 5 & $100 \%$ & 10 & $100 \%$ \\
\hline
\end{tabular}

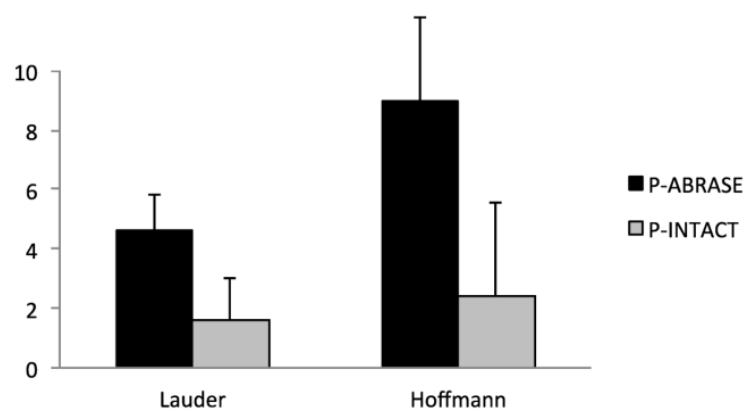

Figure 2: Incidence of cecum-to-mesh adhesions in rats with (P-ABRASE) and without (P-INTACT) cecal abrasion, evaluated with Lauder and total Hoffmann scores. The Lauder scoring scheme for measurement of adhesion formation takes into account number, strength, and distribution of adhesions and counts to a maximum score of 5 , whereas the maximum Hoffmann score is 10 . A combination of polypropylene mesh implantation and a de-peritonealization by cecal abrasion (P-ABRASE) resulted in a significantly increased adhesion score in both scoring systems compared to animals with unimpaired cecal peritoneum (P-INTACT) $(p=0.0002)$ 

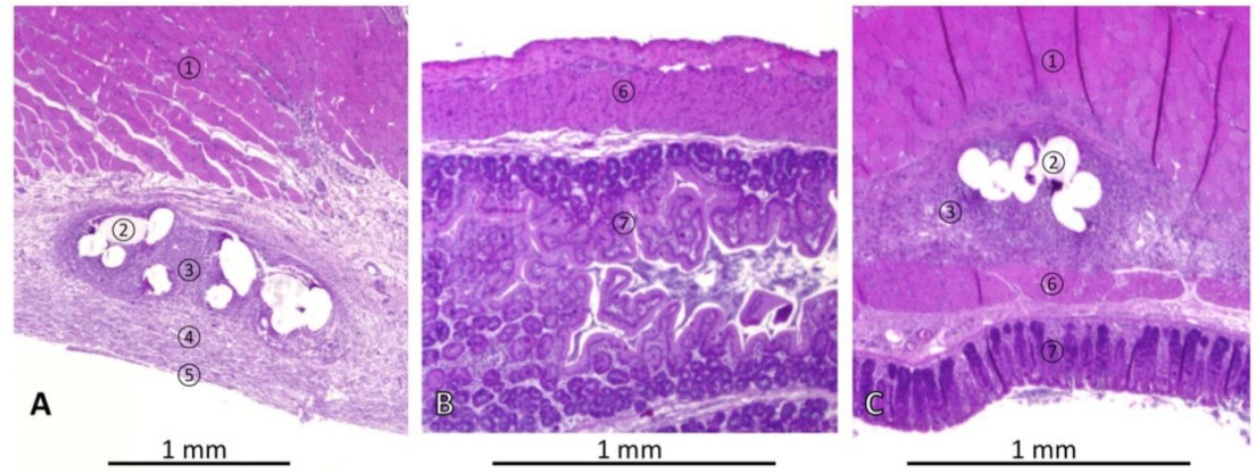

Figure 3: Representative histologies of haematoxylin \& eosin stained tissues of rats with mesh implantation on day 7 autopsy. (A) Abdominal wall of rat without cecal abrasion (P-INTACT), showing skeletal muscle, ingrowth of mesh surrounded by granulating tissue and covered with subperitoneal fibrous tissue and a mesothelial mono-layer. (B) Non-abraded cecum approximated to mesh appears normal. (C) Histopathological finding in a rat with mesh implantation and cecal abrasion (P-ABRASE) with a tight adhesion of ingrown mesh and cecum. (1) = skeletal muscle, (2) = mesh, (3) = granulating tissue, (4) = subperitoneal fibrous tissue, (5) = cellular mono-layer, (6) = muscularis of cecum, (7) = cecal mucosa.

\section{Histology}

Representative histological findings in a rat without cecal abrasion are shown in Figures $3 \mathrm{~A}$ and 3B. The mesh was integrated into the abdominal wall. Fibers of mesh were surrounded by granulating tissue indicating a foreign body reaction covered by loose sub-peritoneal tissue and a mono-layer cell coverage, such as in normal peritoneum (Figure 3A). Specimens of the cecum were sampled next to the area of mesh implantation. These revealed physiological histological structures (Figure 3B). In one representative P-ABRASE rat (Figure $3 \mathrm{C}$ ) the ingrown mesh was directly adhesive to the cecal wall.

\section{Discussion}

The mechanisms of adhesion induction are not yet fully understood. One potential adhesion trigger are foreign bodies e.g. meshes. In several animal studies in rodents, swine and sheep, meshes mostly were in contact to unimpaired visceral and parietal peritoneum ${ }^{23-25}$. Other experimental setups mimic the formation of a hernia sac by creation of an abdominal wall defect 26-29. However, in patients, in which the content of the hernia sac has been dissolved from the hernia wall, another important factor for adhesion formation arises: the dissected areas inevitably will not have a peritoneal coverage. This peritoneal injury can lead to adhesion formation ${ }^{30}$. Adhesions from mesh to the greater omentum occur quite frequently, but seem to be of limited consequence. In contrast, intestine-to-mesh adhesions can lead to severe problems, the most serious ones being fistula formation, infection and bowel obstruction. Hooker et al. ${ }^{31}$ used an animal model for testing adhesion prevention strategies in IPOM surgery with local abrasion of small bowel. Other groups de-peritonealized the cecum to provoke adhesion formation after mesh implantation ${ }^{32,33}$. Since the small intestine and cecum are mobile in the peritoneal cavity, our model additionally places a suture from the cecal mesenterium to the abdominal wall to ensure that mesh and injured peritoneum of abraded cecum stay in contact postoperatively. In addition, by using an uncoated polypropylene mesh, a high probability to induce adhesions could be expected 34 . Consequently, our model imitates the clinical situation of a hernia mesh repair with IPOM combined with former adherent hernia sac content. By this, almost all animals with the uncoated polypropylene mesh and cecum depleted from peritoneum developed severe cecum-to-mesh adhesions within one week. However, rats, in which the peritoneum was left intact, had a low rate of intestine-to-mesh or intraabdominal fat-to-mesh adhesions. Histology revealed a detectable mono-cellular mesothelial layer covering the mesh suggesting a parietal re-peritonealisation as a sign of peritoneal healing within one week. The intraabdominal fat-to-mesh adhesions especially occurred at meshes' edges. We consider these adhesions comparable to omental adhesions in humans, as reported e.g. by Chelala et al. ${ }^{35}$.

Our model with approximation of a polypropylene mesh implant to cecum w/o depletion of visceral peritoneum indicates that the condition of the intestinal surface has a particular impact on formation of intestine-to-mesh adhesions. Since mesh implantation combined with cecal abrasion induces adhesions with high reproducibility, our model is not only suitable for testing different mesh types and coatings, but also other anti-adhesive strategies for their effectiveness to prevent adhesions.

\section{Conclusion}

This study introduces a model mimicking the clinical IPOM situation when mesh comes in contact with normal and with de-peritonealized visceral organs. Even an uncoated polypropylene mesh pro- 
vokes only sparse bowel adhesions as long as the intestinal peritoneum is intact. However, severe adhesions develop with high reproducibility when the peritoneal coverage is missing. This indicates that the integrity of peritoneum is a decisive for bowel to mesh adhesion formation. We suggest our model as a suitable basis for testing mesh types and coatings as well as other agents for their capability to prevent intraperitoneal adhesions.

\section{Competing Interests}

The authors have declared that no competing interest exists.

\section{References}

1. Mueller MD, Tschudi J, Herrmann U, Klaiber C. An evaluation of laparoscopic adhesiolysis in patients with chronic abdominal pain. Surg Endosc 1995;9:802-4.

2. Vrijland WW, Bonthuis F, Steyerberg EW, Marquet RL, Jeekel J, Bonjer HJ. Peritoneal adhesions to prosthetic materials: choice of mesh for incisional hernia repair. Surg Endosc 2000;14:960-3.

3. Holmdahl L, Risberg B, Beck DE, et al. Adhesions: pathogenesis and prevention-panel discussion and summary. Eur J Surg Suppl 1997;577:56-62.

4. Wassenaar EB, Schoenmaeckers EJ, Raymakers JT, Rakic S. Recurrences after laparoscopic repair of ventral and incisional hernia: lessons learned from 505 repairs. Surg Endosc 2009;23:825-32.

5. Franneby U, Gunnarsson U, Wollert S, Sandblom G. Discordance between the patient's and surgeon's perception of complications following hernia surgery. Hernia 2005;9:145-9.

6. Cassar K, Munro A. Surgical treatment of incisional hernia. British journal of surgery 2002;89:534-45.

7. Leber GE, Garb JL, Alexander AI, Reed WP. Long-term Complications Associated With Prosthetic Repair of Incisional Hernias. Arch Surg 1998;133.

8. Bageacu S, Blanc P, Breton C, et al. Laparoscopic repair of incisional hernia: a retrospective study of 159 patients. Surg Endosc 2002;16:345-8.

9. Losanoff JE, Richman BW, Jones JW. Entero-colocutaneous fistula: a late consequence of polypropylene mesh abdominal wall repair: case report and review of the literature. Hernia 2002;6:144-7.

10. Sauerland $S$, Walgenbach $M$, habermalz $B$, Seiler CM, Miserez $M$. Laparoscopic versus open hernia repair. The Cochrane Libraty 2011.

11. Béllon JM, Rodríguez M, Grarcía-Honduvilla N, Pascula G, Gómez V, Buján J. Peritoneal Effects of Prosthetic Meshes Used to Repair Abdominal Wall Defects. Journal of Laparoscopic \& Advanced Surgical Techniques 2007;17.

12. Ellis $\mathrm{H}$. The clinical significance of adhesions: focus on intestinal obstruction. Eur J Surg Suppl 1997:5-9.

13. Wullstein C, Gross E. Laparoscopic compared with conventional treatment of acute adhesive small bowel obstruction. Br J Surg 2003;90:1147-51.

14. Van Der Krabben AA, Dijkstra FR, Nieuwenhuijzen M, Reijnen MM, Schaapveld M, Van Goor H. Morbidity and mortality of inadvertent enterotomy during adhesiotomy. Br J Surg 2000;87:467-71.

15. Parker MC, Wilson MS, van Goor $\mathrm{H}$, et al. Adhesions and colorectal surgery call for action. Colorectal Dis 2007;9 Suppl 2:66-72

16. Halm JA, de Wall LL, Steyerberg EW, Jeekel J, Lange JF. Intraperitoneal polypropylene mesh hernia repair complicates subsequent abdominal surgery. World J Surg 2007;31:423-9; discussion 30

17. Lamber B, Grossi JV, Manna BB, Montes JH, Bigolin AV, Cavazzola LT. May polyester with collagen coating mesh decrease the rate of intraperitoneal adhesions in incisional hernia repair? Arq Bras Cir Dig 2013;26:13-7.

18. Chelala E, Barake H, Estievenart J, Dessily M, Charara F, Alle JL. Long-term outcomes of 1326 laparoscopic incisional and ventral hernia repair with the routine suturing concept: a single institution experience. Hernia 2015.

19. Harris ES, Morgan RF, Rodeheaver GT. Analysis of the kinetics of peritoneal adhesion formation in the rat and evaluation of potential antiadhesive agents. Surgery 1995;117:663-9.

20. Poehnert D, Abbas M, Kreipe HH, Klempnauer J, Winny M. High reproducibility of adhesion formation in rat with meso-stitch approximation of injured cecum and abdominal wall. Int J Med Sci 2015;12:1-6.

21. Lauder CI, Garcea G, Strickland A, Maddern GJ. Use of a modified chitosan-dextran gel to prevent peritoneal adhesions in a rat model. The Journal of surgical research 2011;171:877-82.

22. Hoffmann NE, Siddiqui SA, Agarwal S, et al. Choice of hemostatic agent influences adhesion formation in a rat cecal adhesion model. The Journal of surgical research 2009;155:77-81.

23. Tran H, Saliba L, Chandratnam E, Turingan I, Hawthorne W. Strategies to minimize adhesions to intraperitoneally placed mesh in laparoscopic ventral hernia repair. JSLS 2012;16:89-94.
24. Zinther NB, Wara P, Friis-Andersen H. Intraperitoneal onlay mesh: an experimental study of adhesion formation in a sheep model. Hernia 2010;14:283-9.

25. Marcondes W, Herbella FA, Matone I, Odashiro AN, Goldenberg A. Laparoscopic evaluation of abdominal adhesions with different prosthetic meshes in rabbits. JSLS 2008;12:58-61.

26. Kramer K, Senninger N, Herbst $H$, Probst W. Effective prevention of adhesions with hyaluronate. Arch Surg 2002;137:278-82.

27. Peeters E, van Barneveld KW, Schreinemacher MH, et al. One-year outcome of biological and synthetic bioabsorbable meshes for augmentation of large abdominal wall defects in a rabbit model. J Surg Res 2013;180:274-83.

28. Gaertner WB, Bonsack ME, Delaney JP. Visceral adhesions to hernia prostheses. Hernia 2010;14:375-81.

29. Altinli E, Sumer A, Koksal N, et al. Prevention of adhesion to prosthetic mesh: comparison of oxidized generated cellulose, polyethylene glycol and hylan G-F 20. Ulus Travma Acil Cerrahi Derg 2011;17:377-82.

30. Stommel MW, Strik C, van Goor H. Response to pathological processes in the peritoneal cavity--sepsis, tumours, adhesions, and ascites. Seminars in pediatric surgery 2014;23:331-5.

31. Hooker GD, Taylor BM, Driman DK. Prevention of adhesion formation with use of sodium hyaluronate-based bioresorbable membrane in a rat model of ventral hernia repair with polypropylene mesh--a randomized, controlled study. Surgery 1999;125:211-6.

32. Dinsmore RC, Calton WC, Jr., Harvey SB, Blaney MW. Prevention of adhesions to polypropylene mesh in a traumatized bowel model. J Am Coll Surg 2000;191:131-6.

33. Franconi F, Roux J, Lefebvre-Lacoeuille C, Lemaire L. Imaging visceral adhesion to polymeric mesh using pneumoperitoneal-MRI in an experimental rat model. Surg Endosc 2015;29:1567-73

34. Felemovicius I, Bonsack ME, Hagerman G, Delaney JP. Prevention of adhesions to polypropylene mesh. J Am Coll Surg 2004;198:543-8.

35. Chelala E, Debardemaeker Y, Elias B, Charara F, Dessily M, Allé L. 85 Redo surgeries after 733 laparoscopic treatments for ventral and incisional hernia: Adhesion and recurrence analysis. Hernia 2010;14:123-9. 